\title{
Undifferentiated Sarcoma in the Cerebellopontine Angle of an 11-Year-Old Boy
}

\author{
Jeremy M. Hernandez, Jetan H. Badhiwala, Benedicto C. Baronia, Thorsteinn Gunnarsson, \\ Boleslaw Lach
}

Can J Neurol Sci. 2014; 41: 397-399

Cerebellopontine angle (CPA) tumors account for $5-10 \%$ of intracranial neoplasms in the adult population; by contrast, these lesions constitute only $1 \%$ of pediatric central nervous system (CNS) tumors ${ }^{1}$. In adults, the vast majority (approximately $80 \%$ ) of CPA lesions are vestibular schwannomas (VSs), commonly known as acoustic neuromas. The remainder are largely meningiomas, primary cholesteatomas, schwannomas, epidermoid cysts, ependymomas, other primary tumors of the skull base and inner ear, and metastases. In children, arachnoid cysts, choroid plexus papillomas, gliomas, craniopharyngiomas, and central primitive neuroectodermal tumors (cPNETs) have also been reported in the $\mathrm{CPA}^{1}$. Neoplasms in this CNS region are most commonly of low histological grade. In fact, high-grade extra-axial tumors of the CPA are exceedingly rare and thus carry a substantial risk of being misdiagnosed as benign disease. A high-grade undifferentiated sarcoma involving the CPA has only been described in two previous reports in the literature, both in adult subjects ${ }^{2,3}$. To the authors' knowledge, the present case report is the first to describe a high-grade undifferentiated sarcoma of the cerebellopontine angle affecting a pediatric patient, an 11-year-old boy.

\section{Case Report}

A previously healthy 11-year-old boy presented with a four week history of progressive hearing loss, intermittent headaches, swallowing difficulties, and right-sided facial droop. Physical examination revealed incomplete right-sided facial weakness, without forehead sparing, and complete loss of hearing in the right ear. The remainder of the physical examination was unremarkable.

Magnetic resonance imaging (MRI) of the brain demonstrated a heterogeneously enhancing solid mass with multiple internal foci of hemosiderin deposition, measuring $3.9 \times 3.9 \times 1.9 \mathrm{~cm}$, centered extra-axially within the right CPA cistern (Figure). The mass expanded into the adjacent porus acousticus and completely filled the right internal auditory canal (IAC). In addition, there was subtle enhancement within the cochlea, utricle, and the anterior genu of the facial nerve $(\mathrm{CN}$ VII) at the level of the Gasserian ganglion, as well as the tympanic segment, posterior genu, and mastoid segment, indicating perineural invasion of the temporal bone. There was significant local mass effect, with impingement upon the right lateral aspect of the pons and middle cerebellar peduncle. There was no evidence of downward tonsillar herniation; however, there was a slight upward cerebellar vermis herniation at the level of the tentorial incisura, with associated mild obstructive hydrocephalus of the lateral and third ventricles. Immediately posterior to the solid mass, a $3.4 \times 1.8 \mathrm{~cm}$ extra-axial fluid collection with an internal fluid-blood or fluid-debris level was present, likely representing a tumor-related cyst. These radiological findings suggested a high-grade neoplasm, such as malignant degeneration of a VS or a slow growing ependymal tumor with subsequent hemorrhage. Spinal MRI revealed no abnormalities.

The cerebellopontine angle tumor was resected by a retrosigmoid approach using frameless stereotaxy and a neurosurgical microscope. The dura was opened, revealing a massive cystic structure and a tense, bulging cerebellum. We fenestrated the cyst and then moved onto microsurgical dissection and resection of the primary tumor, which was graybrown, solid, and highly vascular. A carpet of tumor capsule was left behind on the lateral aspect of the mass in order to protect the cranial nerves. No complications occurred during this operative procedure.

Hematoxylin and eosin (HE) stain revealed a 'blue' tumor composed of tightly packed cells with distinct cellular outlines and moderately hyperchromatic nuclei. Many of these neoplastic cells were surrounded by rich reticulin fibers. Nests of tumor cells accumulated around blood vessels and were separated by areas of necrosis and hemorrhage. Apoptotic nuclei were frequent; however, mitotic figures were less obvious. Immunohistochemistry was positive for p53 and vimentin. Furthermore, the tumor had a remarkably high Ki67 index - up to $90 \%$ of the cellular population in some regions - and $100 \%$ immunoreactivity for CD99. By contrast, neoplastic cells were entirely negative for cytokeratin, desmin, actin, placental alkaline phosphatase (PLAP), S100, c-kit, alk-1, alpha fetoprotein (AFP), synaptophysin, beta tubulin, neuron specific

From the Division of Emergency Medicine (JMH), Michael G. DeGroote School of Medicine (JHB), Division of Neurosurgery, Department of Surgery (TG), Department of Pathology and Molecular Medicine (BL), McMaster University, Hamilton, Ontario, Canada; Department of Surgery (BCB), Texas Tech University Health Sciences Center, El Paso, Texas, USA.

Received December 9, 2013. Final Revisions Submitted December 16, 2013. Correspondence to: Jeremy Mark Hernandez, Emergency Medicine, Hamilton General Hospital, Division of Emergency Medicine, 2nd Floor Room 252, 237 Barton Street East, Hamilton, Ontario, L8L 2X2, Canada. Email: jeremy.hernandez@medportal.ca. 

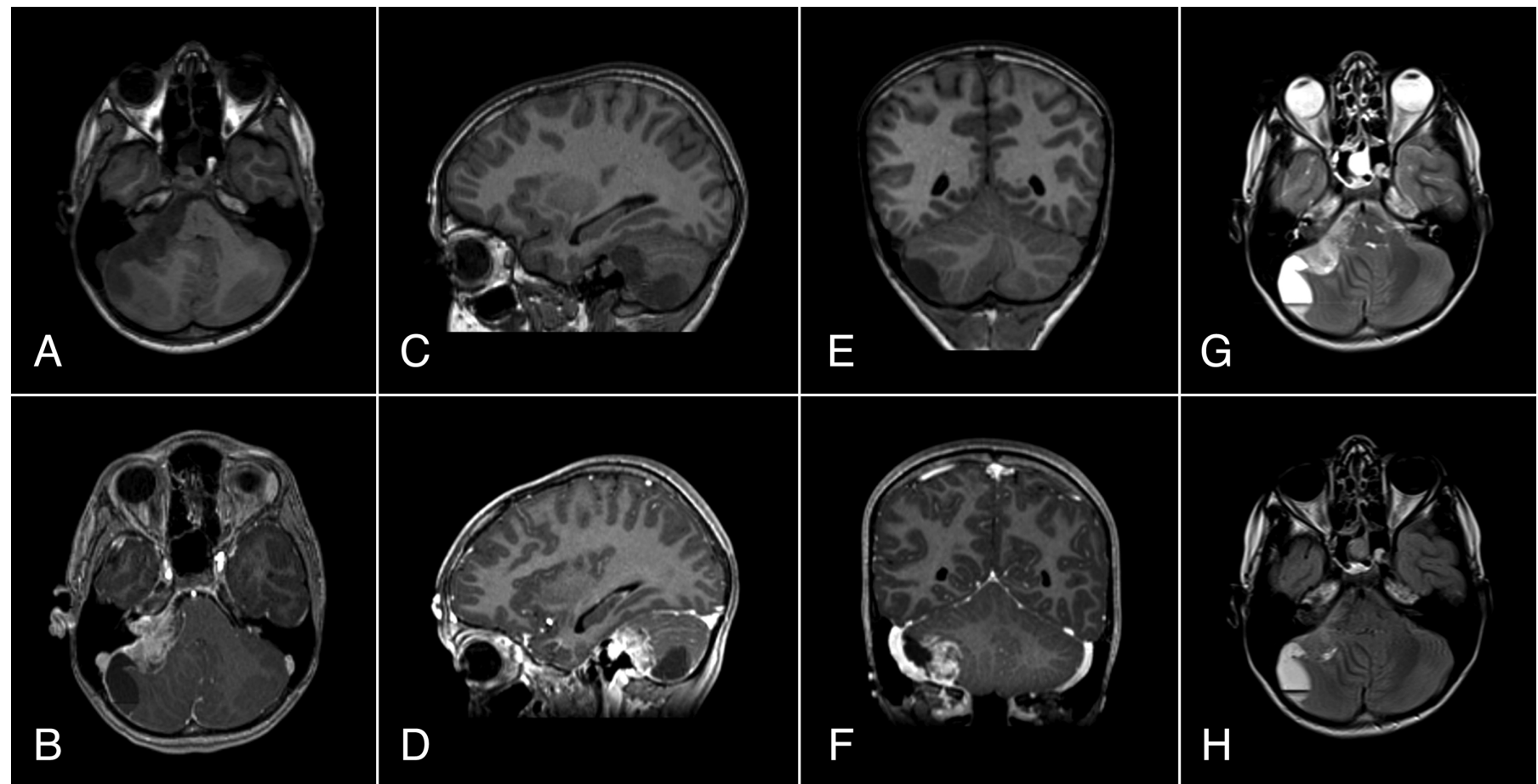

Figure: $(A-F)$ Non-contrast $(A, C, E)$ and post-gadolinium $(B, D, F)$ axial $(A, B)$, sagittal $(C, D)$, and coronal $(E, F)$ T1-weighted MRI head demonstrating a heterogeneously enhancing hemorrhagic right CPA mass with contiguous filling of the IAC. $(G, H)$ Axial T2-weighted $(G)$ and FLAIR (H) MRI head revealing clearly a tumor-related cyst with an internal fluid-blood or fluid-debris level.

enolase, chromogranin, glial fibrilliary acidic protein (GFAP), $\mathrm{CD} 34$, factor 13a, and caldesmon. Immunohistochemistry for INI-1 revealed loss of expression in the nuclei of neoplastic cells. By contrast, control normal cells in the specimen were strongly positive for this antigen. Electron microscopy revealed a pleomorphic tumor with cytoplasm rich in ribosomes, occasional stacks of endoplasmic reticulum, poorly developed Golgi apparatus, and a variable number of mitochondria. There was a significant subpopulation of neoplastic cells containing lakes of glycogen and a variable number of lipid droplets. Nuclei varied from round to very irregular shape with occasional foldings. There was no evidence of formation of any type of intercellular junctions. Occasional cells showed sarcolemmal accumulation of microfilaments. In many areas the background showed viable amounts of collagen and in a single cell, there was an early formation of fibronexus. The background also contained occasional plasma cells undergoing apoptosis and a relatively minimal amount of debris. These histological findings together excluded a neoplasm of neuroectodermal, epithelial, embryonal, rhabdomyosarcomal, or leiomyosarcomal origin. A diagnosis of undifferentiated sarcoma of the CPA was made.

Post-operatively, the patient was neurologically unchanged from pre-operative status, and MRI revealed subtotal resection of the tumor, with no evidence of hemorrhage, hydrocephalus, or cerebral edema. The patient recovered well from surgery and was discharged from hospital on post-operative day four. $\mathrm{He}$ subsequently received four cycles of ifosfamide, carboplatin, etoposide (ICE) chemotherapy, followed by craniospinal irradiation (CSI). Six months after completing therapy (and 13 months following initial presentation), the patient had a relapse of CPA tumor. He eventually succumbed to his disease four months thereafter.

\section{Discussion}

In this report, we describe an undifferentiated sarcoma of the cerebellopontine angle in an 11-year-old boy presenting with symptoms congruent to those seen in lower grade CPA tumors (i.e., VSs, meningiomas, etc.). Our case highlights that both clinical history and physical examination may not be adequate to distinguish this high-grade tumor from more indolent types. There have been only two reports of CPA undifferentiated sarcomas, both occurring in adult patients. In one case, the tumor masqueraded as a vestibular schwannoma. In fact, only microsurgical resection followed by histological examination yielded a definitive diagnosis ${ }^{2}$. The other report described a case where an undifferentiated sarcoma surfaced following surgical resection and stereotactic radiosurgery (SRS) of a VS. The authors underscored the fact that malignant transformation may occur following radiotherapy of VSs, even without an underlying genetic tumor predisposition, such as neurofibromatosis (NF). Such situations should be kept in mind when selecting treatment modalities for a vestibular schwannoma ${ }^{3}$.

In our case, immunohistochemistry revealed a loss of INI-1 expression in neoplastic cells. Loss of expression of INI-1 is commonly found in atypical rhabdoid teratoid tumors (ATRTs). Nonetheless, the present case lacked any immunohistochemical or morphological features of this entity. Notably, previous 
studies have revealed a group of CNS tumors without rhabdoid phenotype that show very aggressive clinical behavior and require especially aggressive therapy. In some of these cases, rhabdoid appearance of neoplastic cells occurred in the recurrence ${ }^{4,5}$.

\section{Conclusion}

A high-grade undifferentiated sarcoma of the CPA is an extremely rare lesion. It has only been described twice in the literature in two adults and never before in children. Although infrequent, based on the present case report, it should be added to the differential diagnosis of CPA lesions in children, because a microsurgical resection followed by histopathological examination remains the only definitive method to differentiate this poor prognosis malignancy from other less aggressive, lower histological grade pathologies presenting in this CNS location.

\section{REFERENCES}

1. Zuccaro G, Sosa F. Cerebellopontine angle lesions in children. Childs Nerv Syst. 2007;23(2):177-83.

2. Carlson ML, Beatty CW, Link MJ. High-grade undifferentiated sarcoma of the cerebellopontine angle masquerading as a benign vestibular schwannoma. Otol Neurotol. 2010;31(8):1350-1.

3. Yang T, Rockhill J, Born DE, Sekhar LN. A case of high-grade undifferentiated sarcoma after surgical resection and stereotactic radiosurgery of a vestibular schwannoma. Skull Base. 2010;20 (3): $179-83$.

4. Haberler C, Laggner U, Slave I, et al. Immunohistochemical analysis of INI1 protein in malignant pediatric CNS tumors: Lack of INI1 in atypical teratoid/rhabdoid tumors and in a fraction of primitive neuroectodermal tumors without rhabdoid phenotype. Am J Surg Pathol. 2006;30(11):1462-8.

5. Kreiger PA, Judkins AR, Russo PA, et al. Loss of INI1 expression defines a unique subset of pediatric undifferentiated soft tissue sarcomas. Mod Pathol. 2009;22(1):142-50. 